\title{
ANALYSING MORTALITY RATES AND DATA OF CHILDHOOD LEUKAEMIA BY TIME SERIES METHODS
}

\author{
M. KIS \\ University of Debrecen, Department of Economic- and Agroinformatics \\ H-4032 Debrecen, Böszörményi út 138. Hungary \\ (phone: +36-52-508-444; fax: +36-52-486-255) \\ e-mail:kiss@thor.agr.unideb.hu \\ (Received $10^{\text {th }}$ Sep 2005, accepted $10^{\text {th }}$ Oct 2006)
}

\begin{abstract}
The Hungarian mortality rates were analyzed by autoregressive integrated moving average models and seasonal time series models examined the data of acute childhood lymphoid leukaemia. The mortality data may be analyzed by time series methods such as autoregressive integrated moving average (ARIMA) modelling. This method is demonstrated by two examples: analysis of the mortality rates of cerebrovascular diseases and analysis of the mortality rates of cancer of cervix. The relationships between time series of mortality rates were studied with ARIMA models. Calculations of confidence intervals for autoregressive parameters by tree methods: standard normal distribution as estimation and estimation of the White's theory and the continuous time case estimation. We present a new approach to analyzing the occurrence of acute childhood lymphoid leukaemia. We decompose time series into components. The periodicity of acute childhood lymphoid leukaemia in Hungary was examined using seasonal decomposition time series method. The seasonal components of the dates of diagnosis revealed that a higher percent of the peaks fell within the winter months than in the other seasons. This proves the seasonal occurrence of the childhood leukaemia in Hungary.
\end{abstract}

Keywords: time series analysis, autoregressive integrated moving average models, mortality rates, seasonal decomposition time series method, acute childhood lymphoid leukaemia

\section{Introduction}

Time series analysis is a well-known method for many years. Box and Jenkins provided a method for constructing time series models in practice [3]. Their method often referred to as the Box-Jenkins approach and the autoregressive integrated moving average models (ARIMA). This method has been applied in the beginning such fields as industry and economics later in medical research as well as $[6,11,10]$.

The method of seasonal time series analysis can be used in various fields of the medicine. With such time series one can detect the periodic trend of the occurrence of a certain disease $[8,7,2]$. Among other diseases, the seasonal periodicity of the childhood lymphoid leukaemia was also analysed using statistical methods [4, 9]. The pathogenesis of the childhood lymphoid leukaemia is still uncertain, but certain environmental effects may provoke the manifestation of latent genes during viral infections, epidemics or pregnancy.

The date of the diagnosis of patients were statistically analysed to determine the role, which the accumulating viral infections and other environmental effects may play during the conception and fatal period on the manifestation of the disease. Because the available data is rather limited and controversial, it seemed logical to make an in-depth analysis of the date of diagnosis of the acute lymphoid leukaemia in Hungarian children. 


\section{Methods}

The Hungarian mortality rates were analysed by autoregressive integrated moving average models. The periodicity of acute childhood lymphoid leukaemia in Hungary was examined using seasonal decomposition time series method.

\section{Autoregressive Moving Average Models}

The mortality data often change in the form of 'time series'. Data of frequencies of mortality rates are usually collected in fixed intervals for several age groups and sexes of the population. Let the value of the mortality rates $z_{t}, z_{t-1}, z_{t-2}, \ldots$ in the years $\mathrm{t}, \mathrm{t}-1, \mathrm{t}-2, \ldots$. For simplicity we assume that the mean value of $\mathrm{z}_{\mathrm{t}}$ is zero, otherwise the $z_{t}$ may be considered as deviations from their mean. Denote $a_{t}, a_{t-1}, a_{t-2}, \ldots$ a sequence of identically distributed uncorrelated random variables with mean 0 and variance $\sigma_{a}{ }^{2}$. The $a_{t}$ are called white noise.

The autoregressive moving average model of order $p, q(\operatorname{ARMA}(p, q))$ can be represent with the following expression $[3,5]$ :

$$
\mathrm{z}_{\mathrm{t}}=\phi_{1} \mathrm{z}_{\mathrm{t}-1}+\ldots+\phi_{\mathrm{p}} \mathrm{z}_{\mathrm{t}-\mathrm{p}}+\mathrm{a}_{\mathrm{t}}+\theta_{1} \mathrm{a}_{\mathrm{t}-1}+\ldots+\theta_{\mathrm{q}} \mathrm{a}_{\mathrm{t}-\mathrm{q}} .
$$

Where $\phi_{1}, \phi_{2}, \ldots, \phi_{\mathrm{p}}$ and $\theta_{1}, \theta_{2}, \ldots, \theta_{\mathrm{q}}$ are parameters, $\mathrm{p}$ means the $\mathrm{p}$ order of autoregressive process and $\mathrm{q}$ denotes the q order of moving average process.

The time series that has a constant mean, variance, and covariance structure, which depends only on the difference between two time points, is called stationary. Many time series are not stationary. It has been found that the series of first differences is often stationary. Let $\mathrm{w}_{\mathrm{t}}$ the series of first differences, $\mathrm{z}_{\mathrm{t}}$ the original time series, than

$$
\mathrm{W}_{\mathrm{t}}=\mathrm{Z}_{\mathrm{t}}-\mathrm{Z}_{\mathrm{t}-1}=\nabla \mathrm{z}_{\mathrm{t}} \text {. }
$$

The Box-Jenkins modelling may be used for stationary time series $[3,5]$. The dependence structure of a stationary time series $z_{t}$ is described by the autocorrelation function: $\rho_{\mathrm{k}}=$ correlation $\left(\mathrm{z}_{\mathrm{t}} \mathrm{z}_{\mathrm{t}+\mathrm{k}}\right)$; $\mathrm{k}$ is called the time lag. This function determines the correlation between $z_{t}$ and $z_{t+k}$. To identify an ARIMA model Box and Jenkins have suggested an iterative procedure [3]:

- for provisional model may be chosen by looking at the autocorrelation function and partial autocorrelation function

- parameters of the model are estimated

- the fitted model is checked

- if the model does not fit the data adequately one goes back to the start and chooses an improved model.

Among different models, which represent the data equally well, one chooses the simplest one, the model with fewest parameters $[3,5]$. The relation between two time series $z_{t}$ and $y_{t}$ can be give by the cross correlation function $\left(\rho_{z y}(k)\right)$; $\rho_{\mathrm{zy}}(\mathrm{k})=$ correlation $\left(\mathrm{z}_{\mathrm{t}} ; \mathrm{y}_{\mathrm{t}+\mathrm{k}}\right)$; where $\mathrm{k}=0, \pm 1, \pm 2, \ldots$. The cross correlation function determines the correlation between the time series as a function of the time lag $\mathrm{k} \mathrm{[3].}$

\section{Estimations for Confidence Intervals}

For estimation the parameter of first order autoregressive model two methods are well known: apply the standard normal distribution as estimation and the White method [1]. These methods cannot be applied in non-stationary case. Little known for estimation of the parameter of first order autoregressive parameter is the application of 
estimation for continuous time case processes [1]. This method can be applied in each case properly.

\section{Seasonal Time Series}

The time series usually consist of three components: the trend, the periodicity and the random effects. The trend is a long-term movement representing the main direction of changes. The periodicity marks cyclic fluctuations within the time series. The irregularity of the peaks and drops form a more-or-less constant pattern around the trend line. Due this stability the length and the amplitude of the seasonal changes is constant or changes very slowly. If the periodic fluctuation pattern is stable, it is called a constant periodic fluctuation. When the pattern changes slowly and regularly over the time, we speak of a changing periodicity. The third component of the time series is the random error causing irregular, unpredictable, non-systematic fluctuations in the data independent from the trend line.

An important part of the time series analysis is the identification and isolation of the time series components. One might ask how these components come together and how can we define the connection between the time series and its components with a mathematical formula. The relationship between the components of a time series can be described either with an additive or a multiplicative model.

Let $y_{i, j}(i=1, \ldots, n ; j=1, \ldots, m)$ marks the observed value of the time series. The index $i$ stands for the time interval (i.e. a year), the $j$ stands for a particular period in the time interval (i.e. a month of the year). By breaking down the time series based on the time intervals and the periods we get a matrix-like table. In the rows of the matrix are the values from the various periods of the same time interval; while in the columns are the values from the same periods over various time intervals.

$$
\begin{gathered}
\mathrm{y}_{1,1} ; \mathrm{y}_{1,2} ; \ldots ; \mathrm{y}_{1, \mathrm{~m}} ; \\
\mathrm{y}_{2,1} ; \mathrm{y}_{2,2} ; \ldots ; \mathrm{y}_{2, \mathrm{~m}} ; \\
\mathrm{y}_{3,1} ; \mathrm{y}_{3,2} ; \ldots ; \mathrm{y}_{3, \mathrm{~m}} ; \\
\mathrm{y}_{\mathrm{n}, 1} ; \mathrm{y}_{\mathrm{n}, 2} ; \ldots ; \mathrm{y}_{\mathrm{n}, \mathrm{m}}
\end{gathered}
$$

Let $d_{i, j}(i=1,2, \ldots, n ; j=1,2, \ldots, m)$ mark the trend of the time series, $\mathrm{s}_{\mathrm{i}, \mathrm{j}} \quad(\mathrm{i}=1,2, \ldots, \mathrm{n} ; \mathrm{j}=1,2, \ldots, \mathrm{m})$, the periodic fluctuation and $\varepsilon_{i, j}(i=1,2, \ldots, n ; j=1,2, \ldots, m)$, the random error. Using these denotations the additive seasonal model can be defined as

$$
\begin{aligned}
& y_{\mathrm{i}, \mathrm{j}}=\mathrm{d}_{\mathrm{i}, \mathrm{j}}+\mathrm{s}_{\mathrm{i}, \mathrm{j}}+\varepsilon_{\mathrm{i}, \mathrm{j}},(\mathrm{i}=1,2, \ldots, \mathrm{n} ; \mathrm{j}=1,2, \ldots, \mathrm{m}) . \\
& \text { the multiplicative model as } \\
& y_{\mathrm{i}, \mathrm{j}}=\mathrm{d}_{\mathrm{i}, \mathrm{j}}{ }^{*} \mathrm{~s}_{\mathrm{i}, \mathrm{j}} * \varepsilon_{\mathrm{i}, \mathrm{j}} ;(\mathrm{i}=1,2, \ldots, \mathrm{n} ; \mathrm{j}=1,2, \ldots, \mathrm{m}) .
\end{aligned}
$$

The trend of a time series can easily be computed with moving averages or analytic trend calculation. Moving averaging generates the trend as the dynamic average of the time series. Analytic trend calculation approximates the long-term movement in the time series with a simple curve (linear, parabolic or exponential curve) and estimates its parameters. The indices of the periodic fluctuation are called seasonal differences (in the additive model) or seasonal ratios (in the multiplicative model). These indices represent the absolute difference from the average of the time interval using the additive model or the percentile difference using the multiplicative model. Seasonal adjustment is done by subtracting the $j$ seasonal difference from the $j$ data value of each $i$ season (additive model) or by dividing the $j$ data value of each $i$ season by the $j$ seasonal ratio 
(multiplicative model). The seasonally adjusted data reflect only the effect of the trend and the random error.

\section{Results}

The SPSS program-package was used for analysing. ARIMA models were identified for some mortality rates. The results are demonstrated two cases from Hungarian mortality rates.

\section{Analysing the Mortality Rates}

The mortality rates of cerebrovascular disease over age 65 for male and female were examined. The autocorrelation functions decay for both data series. The partial autocorrelation functions have a significance value at $k=1$ lag. The first order autoregressive model can be acceptable on the basis of autocorrelation and partial autocorrelation functions. So the stochastic equation over age 65 years of male:

$$
\mathrm{z}_{\mathrm{t}}=0,792 \mathrm{z}_{\mathrm{t}-1}+\varepsilon_{\mathrm{t}} \text {. }
$$

The model over age 65 of female is the following:

$$
\mathrm{Z}_{\mathrm{t}}=0,809 \mathrm{Z}_{\mathrm{t}-1}+\varepsilon_{\mathrm{t}} .
$$

When the fitted model is adequate then the autocorrelation of residuals have $\chi^{2}$ distribution with (K-p-q) degree of freedom [3]. On the basis of test the selected models were adequate because

$$
\chi_{\text {male }}^{2}=1,7478 ; \chi_{\text {female }}^{2}=3,886 ; \chi_{0,05 ; 5}^{2}=11,07 \text {. }
$$

The cross correlation function before fitting model and after fitting model were examined. The function has more significance values before fitting model. The cross correlation function for the residuals has one significance values after fitting model. From behaviour of residuals we may be conclude that between examined time series is "synchronisation". In that years when the mortality rates for male increased the mortality rates for female increased as well.

The change in the mortality rates of cancer of cervix between age class $0-64$ years and over age class 65 years were examined as well. The stochastic equation for the mortality rates for younger age class:

for older age class:

$$
\mathrm{z}_{\mathrm{t}}=0,576 \mathrm{z}_{\mathrm{t}-1}+\varepsilon_{\mathrm{t}} .
$$

$$
\mathrm{z}_{\mathrm{t}}=0,7032 \mathrm{z}_{\mathrm{t}-1}+\varepsilon_{\mathrm{t}} .
$$

On the basis of the $\chi^{2}$ test the selected models were adequate; because

$$
\chi_{0-64}^{2}=1,956 ; \chi^{2} \text { over } 65=2,304 ; \chi_{0,05}^{2}=11,07 \text {. }
$$

The cross correlation function for residuals has not significance value at $\mathrm{k}=0$ lag on $95 \%$ significance level. It may be concluded that there is not "synchronisation" between time series.

The confidence intervals were carried out by three mentioned methods. For the calculations of the confidence limits we used the tables of the known exact distribution of the maximum-likelihood estimator of the damping parameter of an autoregressive process [1]. The confidence intervals for different significance levels for the first order autoregressive parameter of stochastic equation for male of cerebrovascular diseases can be seen in Table 1. 
Table 1. Confidence intervals for different significance levels for the first order autoregressive parameter of stochastic equation for male of cerebrovascular diseases.

\begin{tabular}{l|c|c|c}
\hline \multicolumn{1}{c|}{$\phi \approx \mathbf{0 , 7 9 2}$ (male) } & $\mathbf{p = 0 , 1}$ & $\mathbf{p}=\mathbf{0 , 0 5}$ & $\mathbf{p = 0 , 0 1}$ \\
\hline Normal distribution & $(0,5956 ; 0,9884)$ & $(0,5523 ; 1,0317)$ & $(0,4736 ; 1,1104)$ \\
White method & $(0,5993 ; 0,9847)$ & $(0,5596 ; 1,0244)$ & $(0,4791 ; 1,1105)$ \\
Continuous time process & $(0,6949 ; 0,9424)$ & $(0,6649 ; 0,9723)$ & $(0,6116 ; 0,9978)$ \\
\hline
\end{tabular}

\section{Analysing the Periodicity of Acute Childhood Lymphoid Leukaemia}

The databank of the Hungarian Paediatric Oncology Workgroup contains the data of all the patients with lymphoid leukaemia diagnosed between 1988 and 2000. In this time interval a total of 814 children were registered (of which 467 were boys). The patients were 0-18 years old, with a mean age of 6,4 years and a median of 5,4 years. The components of the time series can be identified and isolated using statistical program packages. The analysis of the seasonal periodicity of the acute childhood lymphoid leukaemia was done with the SPSS statistical program package.

The analysis of the periodicity of acute childhood lymphoid leukaemia was performed on the basis of the date of the diagnosis (year + month) of the disease. We analysed three data series. The first data series contained the number of all the patients diagnosed monthly, the second contained the number of those patients younger than the value of the median, the third contained the number those older than the value of the median.

The seasonal components of all patients revealed 9 peaks. 6 of these peaks fell within the winter months, 1 in the autumn period, 1 in the summer months, 1 in the spring months (Fig. 1). The seasonal components of the younger age group showed 7 peaks in the winter, 1 in the spring and 1 in the summer months. The seasonal components of the older age group showed 7 peaks in the winter, 1 in the spring, 1 in the autumn and 4 in the summer months.

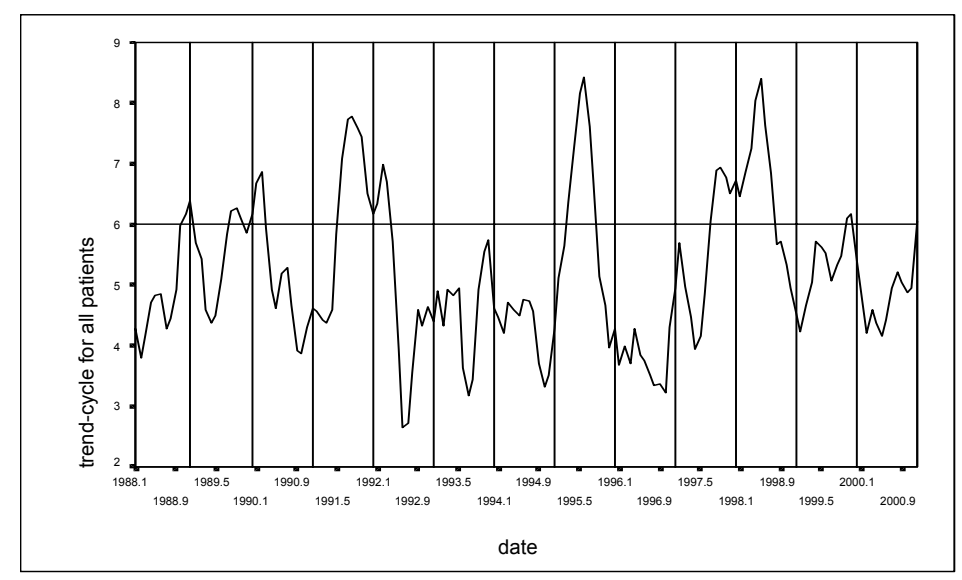

Figure 1. Seasonal components of all patients of acute lymphoid leukaemia diagnosed monthly in the observed period. 


\section{Discussion}

The Box-Jenkins models may be useful for analysing epidemiological time series. The method described the relationships between time series of mortality rates. It reveals strong synchronised behaviour of cerebrovascular diseases between the sexes. For time series of mortality data for cancer of cervix no such synchronisation is found between subgroups.

From the analysis of the first order autoregressive parameters it may be seen that by applying the normal distribution as estimation and White method the confidence intervals are near equal. For the upper estimations of confidence limits we can get larger than one applying these methods. Applying the continuous time process for the estimation of the confidence intervals they are much smaller and it can be used in each case [1].

Analysis of the seasonality of childhood lymphoid leukaemia in Hungary was performed both on the total number of patients and on the data series divided at the median. A certain periodicity was found in the dates of the diagnosis in patients with leukaemia. Although there was some difference in the patterns of the seasonal components peaks of the three time series, the majority of the peaks fell within the winter months in all three-time series. This was more significant in the group of all the patients and in the younger age group. The results of the analyses proved the seasonal occurrence of the childhood lymphoid leukaemia. Some studies reported similar seasonality [13], while other studies denied any kind such periodicity [12]. Our results prove the seasonal occurrence of the childhood lymphoid leukaemia in Hungary. Due to the controversial nature of the available international data, further studies should be carried out.

\section{REFERENCES}

[1] Arato, M., Benczur, A. (1986): Exact distribution of the maximum likelihood estimation for Gaussian-Markovian processes. - In: Tusnady, G., Ziermann, M. (ed.): Time Series Analysis. Technical Publishing House, Budapest.

[2] Barnett, A.G., Dobson, A.J. (2004): Estimating trends and seasonality in coronary heart disease. - Statistics in Medicine 23: 3505-3523.

[3] Box, G.E.R., Jenkins, G.M. (1976): Time Series Analysis, Forecasting and Control. Holden-Day, San Francisco.

[4] Cohen, P. (1987): The influence on survival of onset of childhood acute leukaemia (ALL). - Chronobiology International 4: 291-297.

[5] Csaki, P. (1984): ARMA processes. - In: Tusnady, G., Ziermann, M., (ed.): Time Series Analysis. Technical Publishing House, Budapest.

[6] Diaz, J., Garcia, R., Lopez, C., Linares, C., Tobias, A., Prieto, L. (2005): Mortality impact of extreme temperatures. - International Journal of Biometerology 49: 179-183.

[7] Fung, K.Y., Luginaah, I., Gorey, K.M., Webster, G. (2005): Air pollution and daily hospital admissions for cardiovascular diseases in Windsor, Ontario. - Canadian Journal of Public Health 96: 29-33.

[8] Gallerani, M., Boari, B., de Toma, D., Salmi, R., Manfredini, R. (2004): Seasonal variation in the occurrence of deep vein thrombosis. - Med Sci Monit 10: 191-196.

[9] Harris, R.E., Harrel, F.E., Patil, K.D., Al-Rashid, R. (1987): The seasonal risk of paediatric/childhood acute lymphocyte leukaemia in the United States. - J Chronic Dis 40: 915-923. 
[10] Pereira, A. (2004): Performance of time series methods in forecasting the demand for red blood cell transfusion. - Transfusion 44: 739-746.

[11] Reichert, T.A., Simonsen, L., Sharma, A., Pardo, S.A., Fedson, D.S., Miller, M.A. (2004): Influenza and the winter increase in mortality in the United States, 1959-1999. American Journal of Epidemiology 160: 492-502.

[12] Sorenson, H.T., Pedersen, L., Olse, J.H., et al.: Seasonal variation in month of birth and diagnosis of early childhood acute lymphoblastic leukaemia. - J A M A 285: 168-169.

[13] Vienna, N.J., Polan, A.K. (1976): Childhood lymphatic leukaemia prenatal seasonality and possible association with congenital Varicella. - American Journal of Epidemiology 103: 321-332. 